\title{
LIMIT POINTS OF COMMUTING SQUARES
}

\author{
REMUS NICOARA \\ UNIVERSITY OF TENNESSEE, KNOXVILLE
}

\begin{abstract}
In an attempt to understand the structure of the moduli space of commuting squares, we ask the question: when is a commuting square $\mathfrak{C}$ a limit of non-isomorphic commuting squares? We present necessary second order conditions on such a $\mathfrak{C}$.

We give an application to the classification of complex Hadamard matrices. Such matrices correspond to spin model commuting squares. We exemplify on Petrescu's matrices how our result can be used to decide if a one-parameter family can be extended to a multi-parametric family of Hadamard matrices.
\end{abstract}

\section{INTRODUCTION}

Commuting squares were introduced by S. Popa in [Po1] (see also [Po2], [JS]). They arise naturally in subfactor theory, as invariants and construction data for subfactors. A commuting square is a square of inclusions of finite dimensional von Neumann algebras:

$$
\mathfrak{C}=\left(\begin{array}{ccc}
P_{-1} & \subset & P_{0} \\
\cup & & \cup, \tau \\
Q_{-1} & \subset & Q_{0}
\end{array}\right)
$$

with a faithful trace $\tau$ on $P_{0}$, such that the vector spaces $P_{-1} \ominus Q_{-1}$ and $Q_{0} \ominus Q_{-1}$ are orthogonal with respect to the inner product defined by $\tau$ on $P_{0}$.

Commuting squares show up in the standard invariant of a subfactor and are complete invariants for a large class of subfactors, those of finite depth (see [Po1]).

Conversely, one can construct from a commuting square a finite index hyperfinite subfactor, by iterating Jones' basic construction ([Jon]). Most of the known explicit examples of subfactors were obtained using this construction. One interest in the existence of (continuous) families of commuting squares is to obtain families of possibly non-isomorphic subfactors, constructed from the commuting squares.

In $[\mathrm{Ni}]$ we introduced a condition for arbitrary commuting squares $\mathfrak{C}$, called the span condition, as a maximality condition for the dimension of the commutator $\left[P_{-1}, Q_{0}\right]=\left\{p q-q p, p \in P_{-1}, q \in Q_{0}\right\}$. We proved 
that every commuting square satisfying the span condition is isolated among all commuting squares, modulo isomorphisms. The main technique we used was taking what one might call the first order derivative of the commuting square relation, along a direction of convergence.

Consequently, if a commuting square $\mathfrak{C}$ is not isolated then the span condition must fail. In this paper we find a new restriction that $\mathfrak{C}$ must satisfy if it is not isolated. It states that a certain map in two variables $(p, q) \in P_{-1} \times Q_{0}$ actually depends only on the commutator $[p, q]=$ $p q-q p$ (Corollary 3.3). This result is obtained by taking the "second order derivative" of the commuting square relations. The rigidity of the commuting square relations allows us to overcome some technical difficulties regarding the convergence of the second order terms.

In the last section of the paper we present some applications to commuting squares arising from complex Hadamard matrices (see [Po3]). In the recent years, complex Hadamard matrices have found applications in several areas of mathematics and physics, such as quantum information theory, error correcting codes, spectral sets and Fuglede's conjecture. The classification of Hadamard matrices is largely unknown (see $[\mathrm{TaZy}])$.

Our result can be used to show that certain parametric families of Hadamard matrices can not be extended to multi-parametric families. We exemplify on Petrescu's matrices of order 7 ([Pe]). These are the only known infinite families of Hadamard matrices of order 7 . They consist in two one-parameter families, intersecting at one point. It is a natural question in the study of the moduli space of Hadamard matrices whether these families are in fact part of a 2-dimensional manifold. We answer it in the negative. 


\section{Preliminaries}

In this section we recall the definition of isolation in the context of commuting squares, as well as the span condition and some other results from [Ni]. All algebras considered will be matrix algebras, i.e. *-closed subalgebras of $\mathbb{M}_{n}(\mathbb{C})$ for some $n \geq 1$. Such an algebra is always of the form $\oplus_{i} \mathbb{M}_{n_{i}}(\mathbb{C})$, with $n_{i}$ positive integers.

We recall the definition of a commuting square (see [Po2]):

Definition 2.1. A commuting square of matrix algebras is a square of inclusions:

$$
\left(\begin{array}{ccc}
P_{-1} & \subset & P_{0} \\
\cup & & \cup, \tau \\
Q_{-1} & \subset & Q_{0}
\end{array}\right)
$$

where $P_{0}, P_{-1}, Q_{0}, Q_{-1}$ are matrix algebras and $\tau$ a faithful positive trace on $P_{0}, \tau(1)=1$, satisfying the condition:

$$
E_{P_{-1}} E_{Q_{0}}=E_{Q_{-1}}
$$

Here $E_{A}=E_{A}^{P_{0}}$ denotes the $\tau$-invariant conditional expectation of $P_{0}$ onto the subalgebra $A \subset P_{0}$.

We say that the commuting square is non-degenerate if $P_{0}=$ $\operatorname{span}\left(P_{-1} \cdot Q_{0}\right)$.

We will assume all our commuting squares to be non-degenerate.

Definition 2.2. We say that the commuting squares

$$
\mathfrak{C}=\left(\begin{array}{ccc}
P_{-1} & \subset & P_{0} \\
\cup & & \cup, \tau \\
Q_{-1} & \subset & Q_{0}
\end{array}\right), \tilde{\mathfrak{C}}=\left(\begin{array}{ccc}
\tilde{P}_{-1} & \subset & \tilde{P}_{0} \\
\cup & & \cup, \tilde{\tau} \\
\tilde{Q}_{-1} & \subset & \tilde{Q}_{0}
\end{array}\right)
$$

are isomorphic if there exists a trace-invariant ${ }^{*}$-isomorphism $\phi: P_{0} \rightarrow$ $\tilde{P}_{0}$ such that $\phi\left(P_{-1}\right)=\tilde{P}_{-1}, \phi\left(Q_{-1}\right)=\tilde{Q}_{-1}, \phi\left(Q_{0}\right)=\tilde{Q}_{0}$

The following definition is from [Chr]:

Definition 2.3. Let $A$ be a matrix algebra with normalized trace $\tau$. Denote $\mathcal{S}(A)=$ the set of all *-subalgebras of $A$ containing the identity. For $B_{1}, B_{2} \in \mathcal{S}(A)$ and $\delta>0$ we say that $B_{1}$ is $\delta$-contained in $B_{2}$ if for every element $x \in B_{1}$ of $\|x\|=1$ there exists $y \in B_{2}$ such that $\|x-y\|_{2}<\delta$. If $B_{1}$ is $\delta$-contained in $B_{2}$ and $B_{2}$ is $\delta$-contained in $B_{1}$ we write $\left\|B_{1}-B_{2}\right\|_{2, A}<\delta$.

We can now recall the definition of isolation from $[\mathrm{Ni}]$ : 
Definition 2.4. The commuting square of matrix algebras

$$
\mathfrak{C}=\left(\begin{array}{ccc}
P_{-1} & \subset & P_{0} \\
\cup & & \cup, \tau \\
Q_{-1} & \subset & Q_{0}
\end{array}\right)
$$

is isolated (among all commuting squares) if there exists $\delta>0$ such that if

$$
\tilde{\mathfrak{C}}=\left(\begin{array}{ccc}
\tilde{P}_{-1} & \subset & \tilde{P}_{0} \\
\cup & & \cup, \tilde{\tau} \\
\tilde{Q}_{-1} & \subset & \tilde{Q}_{0}
\end{array}\right)
$$

is a commuting square and $\phi: P_{0} \rightarrow \tilde{P}_{0}$ a trace-invariant $*$ isomorphism satisfying

$\left\|\phi\left(P_{-1}\right)-\tilde{P}_{-1}\right\|_{2, \tilde{P}_{0}}<\delta,\left\|\phi\left(Q_{-1}\right)-\tilde{Q}_{-1}\right\|_{2, \tilde{P}_{0}}<\delta,\left\|\phi\left(Q_{0}\right)-\tilde{Q}_{0}\right\|_{2, \tilde{P}_{0}}<\delta$

then $\tilde{\mathfrak{C}}$ is isomorphic to $\mathfrak{C}$.

In $[\mathrm{Ni}]$ we showed that to check isolation it is enough to restrict to commuting squares in which just one corner changes:

Lemma 2.5. Let

$$
\mathfrak{C}=\left(\begin{array}{ccc}
P_{-1} & \subset & P_{0} \\
\cup & & \cup, \tau \\
Q_{-1} & \subset & Q_{0}
\end{array}\right)
$$

be a commuting square of finite dimensional von Neumann algebras, with trace $\tau . \mathfrak{C}$ is isolated if and only if there exists $\varepsilon>0$ such that if $U \in Q_{-1}^{\prime} \cap P_{0}$ is a unitary, $\|U-I\|_{2}<\varepsilon$, and

$$
\mathfrak{C}(U)=\left(\begin{array}{ccc}
P_{-1} & \subset & P_{0} \\
\cup & & \cup \\
Q_{-1} & \subset & U Q_{0} U^{*}
\end{array}\right)
$$

is a commuting square, then $\mathfrak{C}(U)$ is isomorphic to $\mathfrak{C}$.

Assume $\mathfrak{C}$ is not isolated. This means that there exists a sequence of unitaries $U_{n} \rightarrow I$ in $Q_{-1}^{\prime} \cap P_{0}$, such that $\mathfrak{C}\left(U_{n}\right)$ is not isomorphic to $\mathfrak{C}, \forall n \geq 1$.

We can write $U_{n}$ as $U_{n}=\exp \left(i h_{n}\right)$ with $h_{n} \in Q_{-1}^{\prime} \cap P_{0}$ hermitian non-zero elements, converging to 0 . Because of the compactness of the unit ball of the finite dimensional algebra $P_{0}$, by eventually passing to a subsequence of $h_{n}$ we may assume that $\frac{h_{n}}{\left\|h_{n}\right\|} \rightarrow h \in Q_{-1}^{\prime} \cap P_{0},\|h\|=1$. It is easy to see that $h=\lim _{n \rightarrow \infty} \frac{U_{n}-I}{i\left\|U_{n}-I\right\|}$.

Definition 2.6. $h$ is called the direction of convergence of the sequence $\mathfrak{C}\left(U_{n}\right)$. 
In [Ni] it was proven that the unitaries $U_{n}$ can be modified, without altering the isomorphism equivalence class of the commuting squares, such that $h$ also satisfies: $E_{P_{-1}^{\prime} \cap P_{0}}(h)=E_{Q_{0}^{\prime} \cap P_{0}}(h)=E_{Q_{-1}^{\prime} \cap P_{-1}}(h)=$ $E_{Q_{-1}^{\prime} \cap Q_{0}}(h)=0$.

We will refer to such an $h$ as a normalized direction of convergence.

We give some notations needed for the span condition.

Notation 2.7. Let $\mathfrak{C}$ be a commuting square as before. For $V, W$ vector subspaces of the algebra $P_{0}$, denote

$$
\begin{gathered}
V+W=\{v+w \mid v \in V, w \in W\} \\
{[V, W]=\operatorname{span}\{v w-w v \mid v \in V, w \in W\}}
\end{gathered}
$$

Denote by $H(\mathfrak{C})$ the following vector subspace of $P_{0}$, which we will refer to as the spanning space of $\mathfrak{C}$ :

$H(\mathfrak{C})=\left[P_{-1}, Q_{0}\right]+\left(Q_{-1}^{\prime} \cap P_{-1}\right)+\left(Q_{-1}^{\prime} \cap Q_{0}\right)+\left(P_{-1}^{\prime} \cap P_{0}\right)+\left(Q_{0}^{\prime} \cap P_{0}\right)$

Definition 2.8. $\mathfrak{C}$ is said to satisfy the span condition if $H(\mathfrak{C})=P_{0}$.

We end this section by recalling the main result of $[\mathrm{Ni}]$ :

Theorem 2.9. If $\mathfrak{C}$ is not isolated and $h$ is a normalized direction of convergence for $\mathfrak{C}$ then $h$ satisfying the conditions:

(i). $h=h^{*}$

(ii). $\tau(h[p, q])=0$ for all $p \in P_{-1}$ and $q \in Q_{0}$.

In other words, any direction of convergence $h$ is a hermitian orthogonal to the vector space $H(\mathfrak{C})$.

Corollary 2.10. If $\mathfrak{C}$ satisfies the span condition then $\mathfrak{C}$ is isolated.

\section{The Main Result}

We have seen that if $H(\mathfrak{C})=P_{0}$ then the commuting square $\mathfrak{C}$ is isolated. We now ask the reverse question: assume there exists a hermitian $h \in P_{0} \ominus H(\mathfrak{C}),\|h\|=1$. Does there exist a sequence of unitaries $U_{n} \rightarrow I$ of direction $h, U_{n} \in P_{0}$, such that $\mathfrak{C}\left(U_{n}\right)$ are all non-isomorphic commuting squares?

We find new restrictions on $h$ for the existence of such a sequence of commuting squares. The span condition was found by taking the "derivative" of the commuting square relation along the direction $h$. Our new conditions are obtained by considering what one might call the second derivative along $h$. The main technical difficulty is using the commuting square and unitary relations to control the growth of the second order coefficient of $U_{n}$ in terms of the first order coefficient. 
In the next theorem we will often use the following relation that holds true for every $a, b, c \in P_{0}$ :

$$
\tau([a, b] c)=\tau(a[b, c])=\tau([c, a] b)
$$

as it can easily be checked: $\tau([a, b] c)=\tau(a b c-b a c)=\tau(a b c)-\tau(b a c)=$ $\tau(a b c)-\tau(a c b)=\tau(a[b, c])=\tau(c a b)-\tau(a c b)$

Theorem 3.1. Let

$$
\mathfrak{C}=\left(\begin{array}{ccc}
P_{-1} & \subset & P_{0} \\
\cup & & \cup, \tau \\
Q_{-1} & \subset & Q_{0}
\end{array}\right)
$$

be a commuting square of finite dimensional von Neumann algebras, with trace $\tau$. If $\mathfrak{C}$ is not isolated and $h$ is a normalized direction of convergence then there exists $s \in P_{0}$ satisfying the conditions:

(i). $s+s^{*}=h^{2}$

(ii). $\tau(s[p, q])=\tau\left(p q h^{2}\right)-\tau(p h q h)$ for all $p \in P_{-1}$ and $q \in Q_{0}$.

Remark 3.2. Condition $(i)$ is just a normalization on $s$, as will result from the proof. Condition (ii) entails the new restriction on $h$, which states that the bilinear map $(p, q) \rightarrow \tau\left(p q h^{2}\right)-\tau(p h q h)$ depends just on $[p, q]$.

Proof. Let $U_{n} \in Q_{-1}^{\prime} \cap P_{0}$ be unitaries converging to the identity and of direction $h$, i.e. $h=\lim _{n \rightarrow \infty} \frac{U_{n}-I}{i\left\|U_{n}-I\right\|}$, such that $\mathfrak{C}\left(U_{n}\right)$ are commuting squares non-isomorphic to $\mathfrak{C}$. We know from Theorem 2.9 that $h$ is orthogonal to $\left[P_{-1}, Q_{0}\right]$.

Let $c_{n}=\left\|U_{n}-I\right\|$ and let $v_{n}=\frac{U_{n}-I}{i c_{n}}-h$, for all $n \geq 1$. Clearly $v_{n}$ converges to 0 and $U_{n}=I+i c_{n} h+i c_{n} v_{n}$.

We express the unitary condition $U_{n} \cdot U_{n}^{*}=I$ in terms of $v_{n}$ :

$I=\left(I+i c_{n} h+i c_{n} v_{n}\right)\left(I-i c_{n} h-i c_{n} v_{n}^{*}\right)=I+i c_{n}\left(v_{n}-v_{n}^{*}\right)+c_{n}^{2}\left(h^{2}+\right.$ $\left.h v_{n}+v_{n} h+v_{n} v_{n}^{*}\right)$. After canceling $I$ and dividing by $c_{n}^{2}$ we obtain:

$$
\frac{i\left(v_{n}-v_{n}^{*}\right)}{c_{n}}=-\left(h+v_{n}\right)\left(h+v_{n}^{*}\right)
$$

In particular this means that

$$
\lim _{n \rightarrow \infty} \frac{i\left(v_{n}-v_{n}^{*}\right)}{c_{n}}=-h^{2}
$$

Let us now use the commuting square relations. Let $p \in P_{-1} \ominus Q_{-1}$ and $q \in Q_{0}$. Since $\mathfrak{C}\left(U_{n}\right)$ is a commuting square we now that $p$ is orthogonal to $U_{n} q U_{n}^{*}$ for all $n$. Thus:

$$
\tau\left(p U_{n} q U_{n}^{*}\right)=0, \forall n \geq 1
$$


We rewrite this relation in terms of $v_{n}$ :

$$
\tau\left(p\left(I+i c_{n} h+i c_{n} v_{n}\right) q\left(I-i c_{n} h-i c_{n} v_{n}^{*}\right)\right)=0
$$

After opening the parenthesis and using $\tau(p q)=0$ we obtain: $c_{n} \tau\left(-i p q h+i p h q-i p q v_{n}^{*}+i p v_{n} q\right)+c_{n}^{2} \tau\left(p h q h+p h q v_{n}^{*}+p v_{n} q h+p v_{n} q v_{n}^{*}\right)=$ 0 .

We have $\tau(h[p, q])=0$ implies $\tau(-i p q h+i p h q)=\tau(-i p q h)+$ $\tau(i p h q)=\tau(-i p q h)+\tau(q i p h)=i \tau((-p q+q p) h)=0$. Thus the precedent relation becomes: $c_{n} \tau\left(-i p q v_{n}^{*}+i p v_{n} q\right)+c_{n}^{2} \tau\left(p h q h+p h q v_{n}^{*}+\right.$ $\left.p v_{n} q h+p v_{n} q v_{n}^{*}\right)=0$. After dividing by $c_{n}^{2}$ and taking the limit we obtain:

$$
\lim _{n \rightarrow \infty} \tau\left(\frac{-i p q v_{n}^{*}+i p v_{n} q}{c_{n}}\right)=-\tau(p h q h)
$$

for all $p \in P_{-1} \ominus Q_{-1}$ and $q \in Q_{0}$.

Since $\tau\left(\frac{-i p q v_{n}^{*}+i p v_{n} q}{c_{n}}\right)=\tau\left(\frac{i p q\left(v_{n}-v_{n}^{*}\right)}{c_{n}}\right)+\tau\left(\frac{-i p q v_{n}+i p v_{n} q}{c_{n}}\right)$ and $\lim _{n \rightarrow \infty} \frac{i\left(v_{n}-v_{n}^{*}\right)}{c_{n}}=-h^{2}$ we obtain:

$$
\lim _{n \rightarrow \infty} \tau\left(\frac{-i p q v_{n}+i p v_{n} q}{c_{n}}\right)=-\tau(p h q h)+\tau\left(p q h^{2}\right)
$$

Equivalently, after using $\tau\left(p v_{n} q\right)=\tau\left(q p v_{n}\right)$ :

$$
\lim _{n \rightarrow \infty} \tau\left([p, q] \frac{v_{n}}{i c_{n}}\right)=-\tau(p h q h)+\tau\left(p q h^{2}\right)
$$

We know that $(3)$ holds for all $(p, q) \in\left(P_{-1} \ominus Q_{-1}\right) \times Q_{0}$. We want to show that it actually holds for all $(p, q) \in P_{-1} \times Q_{0}$. By linearity it is sufficient to show that it also holds for $(p, q) \in Q_{-1} \times Q_{0}$. The right side is clearly 0 as $h$ commutes with $p \in Q_{-1}$. The left side is also 0 since $\tau\left([p, q] v_{n}\right)=\tau\left(\left[v_{n}, p\right] q\right)$ and $\left[v_{n}, p\right]=0$ if $p \in Q_{-1}$. Here we used that $U_{n} \in Q_{-1}^{\prime} \cap P_{0}$, which implies $v_{n} \in Q_{-1}^{\prime} \cap P_{0}$.

Note that we do not know if the sequence $\frac{v_{n}}{i c_{n}}$ is bounded, which would allow to pass to a convergent subsequence whose limit $s$ would satisfy the theorem.

Let $s_{n}$ be the orthogonal projection of $\frac{v_{n}}{i c_{n}}$ onto the subspace $\left[P_{-1}, Q_{0}\right]$ of $P_{0}$. The projection is taken with respect to the inner product of $P_{0}$ given by the trace $\tau:\langle x, y\rangle=\tau\left(y^{*} x\right)$. We thus have $\tau\left([p, q] \frac{v_{n}}{i c_{n}}\right)=$ $\tau\left([p, q] s_{n}\right)$ for all $n \geq 1$. Relation (3) becomes:

$$
\lim _{n \rightarrow \infty} \tau\left([p, q] s_{n}\right)=-\tau(p h q h)+\tau\left(p q h^{2}\right)
$$

Note that $\left[P_{-1}, Q_{0}\right]$ is *-closed, as $[p, q]^{*}=-\left[p^{*}, q^{*}\right]$, and thus $v^{*} \in\left[P_{-1}, Q_{0}\right]$ whenever $v \in\left[P_{-1}, Q_{0}\right]$. It follows that the sequence $<s_{n}, v>=\tau\left(v^{*} s_{n}\right)$ converges for all $v \in\left[P_{-1}, Q_{0}\right]$. Thus $s_{n}$ can be thought of as a sequence of vectors in the Hilbert space $\left[P_{-1}, Q_{0}\right]$ whose 
coefficients (in some orthonormal basis of $\left[P_{-1}, Q_{0}\right]$ ) converge. It follows from finite dimensionality that $s_{n}$ converges to some $s_{0} \in\left[P_{-1}, Q_{0}\right]$ and $s_{0}$ satisfies:

$$
\tau\left([p, q] s_{0}\right)=-\tau(p h q h)+\tau\left(p q h^{2}\right)
$$

By taking the adjoint of (4) and using the properties of the trace $\tau$ we obtain:

$$
\tau\left(-\left[p^{*}, q^{*}\right] s_{0}^{*}\right)=-\tau\left(p^{*} h q^{*} h\right)+\tau\left(q^{*} p^{*} h^{2}\right)
$$

Since $p^{*}, q^{*}$ are generic elements of $P_{-1}$ respectively $Q_{0}$ we also have:

$$
\tau\left(-[p, q] s_{0}^{*}\right)=-\tau(p h q h)+\tau\left(q p h^{2}\right)
$$

for all $p \in P_{-1}$ and $q \in Q_{0}$. After subtracting this relation from (4) we obtain:

$$
\tau\left([p, q]\left(s_{0}+s_{0}^{*}\right)\right)=\tau\left([p, q] h^{2}\right)
$$

Thus $s_{0}+s_{0}^{*}-h^{2}$ is orthogonal to $\left[P_{-1}, Q_{0}\right]$. Let $s=s_{0}-\frac{1}{2}\left(s_{0}+s_{0}^{*}-h^{2}\right)$. Then $s$ still satisfies $(4)$, as $\tau([p, q] s)=\tau\left([p, q] s_{0}\right)$. We also have $s+s^{*}=$ $h^{2}$. Thus both relations $(i)$ and $(i i)$ are satisfied.

Corollary 3.3. If the commuting square $\mathfrak{C}$ is not isolated then there exists a hermitian $h \in P_{0}$ such that:

(i). $h$ is orthogonal to $H(\mathfrak{C})=\left[P_{-1}, Q_{0}\right]+\left(Q_{-1}^{\prime} \cap P_{-1}\right)+\left(Q_{-1}^{\prime} \cap\right.$ $\left.Q_{0}\right)+\left(P_{-1}^{\prime} \cap P_{0}\right)+\left(Q_{0}^{\prime} \cap P_{0}\right)$

(ii). The bilinear map on $P_{-1} \times Q_{0}$ given by $(p, q) \rightarrow \tau\left(p q h^{2}\right)-$ $\tau($ phqh $)$ depends only on $[p, q]$. More precisely, whenever $p_{i} \in P_{-1}, q_{i} \in$ $Q_{0}(1 \leq i \leq n$ for some $n \geq 1)$ satisfy $\sum_{i=1}^{n}\left[p_{i}, q_{i}\right]=0$, we must have $\sum_{i=1}^{n} \tau\left(p_{i} q_{i} h^{2}\right)-\tau\left(p_{i} h q_{i} h\right)=0$.

Remark 3.4. From $(i)$ we know that if $\mathfrak{C}$ is not isolated then $H(\mathfrak{C}) \neq$ $P_{0}$. Moreover, it is easy to check that $\left[P_{-1}, Q_{0}\right]$ is orthogonal to $\left(Q_{-1}^{\prime} \cap\right.$ $\left.P_{-1}\right)+\left(Q_{-1}^{\prime} \cap Q_{0}\right)+\left(P_{-1}^{\prime} \cap P_{0}\right)+\left(Q_{0}^{\prime} \cap P_{0}\right)$. Thus condition (ii) is meaningful, as the vector space $\left[P_{-1}, Q_{0}\right]$ has dimension significantly smaller than $\operatorname{dim}\left(P_{-1}\right) \cdot \operatorname{dim}\left(Q_{0}\right)=\operatorname{dim}\left(P_{0}\right)$.

\section{EXAMPLES}

In this section we show that the new restriction $(i i)$ found on $h$ is meaningful and it does not happen automatically for any direction $h$ orthogonal to $H(\mathfrak{C})$. We restrict ourselves to commuting squares arising from complex Hadamard matrices, i.e. we assume $\mathfrak{C}$ to be of the form:

$$
\mathfrak{C}=\left(\begin{array}{ccc}
\mathcal{D}_{n} & \subset & M_{n}(\mathbb{C}) \\
\cup & & \cup \\
\mathbb{C} & \subset & U \mathcal{D}_{n} U^{*}
\end{array}\right)
$$


where $D_{n}$ denotes the algebra of diagonal matrices of size $n$ and $U$ is a unitary matrix with all entries of absolute value $\frac{1}{\sqrt{n}}$. This condition on $U$ is equivalent to $\mathfrak{C}$ being a commuting square (see [Po3], [Ni]). In other words $U=\frac{1}{\sqrt{n}} A$, where $A$ is a complex Hadamard matrix.

Let $p_{1}, p_{2}, \ldots, p_{n}$ be the diagonal matrix units of order $n$ and let $q_{i}=U p_{i} U^{*}$ for $1 \leq i \leq n$. The space $\left[P_{-1}, Q_{0}\right]=\left[\mathcal{D}_{n}, U \mathcal{D}_{n} U^{*}\right]$ is thus spanned by $\left[p_{i}, q_{j}\right], 1 \leq i, j \leq n$. Hence $H(\mathcal{C})$ is spanned by $p_{i}, q_{j},\left[p_{i}, q_{j}\right], 1 \leq i, j \leq n$.

Assume that $\mathcal{C}$ is not isolated and let $h$ be any normalized direction of convergence for $\mathcal{C}$. According to Theorem 3.1, there exists a matrix $s$ such that

$$
\tau\left(s\left[p_{i}, q_{j}\right]\right)=\tau\left(p_{i} q_{j} h^{2}\right)-\tau\left(p_{i} h q_{j} h\right) \text { for all } 1 \leq i, j \leq n
$$

This is equivalent to asking for a linear system of $n^{2} \times n^{2}$ equations with $n^{2}$ unknowns (the entries of $s$ ) to be consistent. We give a concrete example when the system is not consistent for some $h$ perpendicular to the space $H(\mathcal{C})$.

Let $\lambda \in \mathbb{T}, w=\cos \frac{2 \pi}{6}+i \sin \frac{2 \pi}{6}$ and let $U(\lambda)$ denote the following family of complex Hadamard matrices found by Petrescu in [Pe]:

$$
U(\lambda)=\frac{1}{\sqrt{7}}\left(\begin{array}{ccccccc}
\lambda w & \lambda w^{4} & w^{5} & w^{3} & w^{3} & w & 1 \\
\lambda w^{4} & \lambda w & w^{3} & w^{5} & w^{3} & w & 1 \\
w^{5} & w^{3} & \bar{\lambda} w & \bar{\lambda} w^{4} & w & w^{3} & 1 \\
w^{3} & w^{5} & \bar{\lambda} w^{4} & \bar{\lambda} w & w & w^{3} & 1 \\
w^{3} & w^{3} & w & w & w^{4} & w^{5} & 1 \\
w & w & w^{3} & w^{3} & w^{5} & w^{4} & 1 \\
1 & 1 & 1 & 1 & 1 & 1 & 1
\end{array}\right)
$$

For the purpose of calculations, it is better to express $U(\lambda)$ by using the following formula:

$$
U(\lambda)=\left(I+(\lambda-1) p q+(\bar{\lambda}-1) p^{\prime} q^{\prime}\right) U
$$

where $p=p_{2}+p_{3}, p^{\prime}=p_{4}+p_{5}, q=U p U^{*}, q^{\prime}=U q U^{*}$ (see $\left.[\mathrm{Ni}]\right)$.

Let $\mathfrak{C}$ be the commuting square constructed from $U=U(1)$. Clearly $\mathfrak{C}$ is not isolated, as $U(\lambda) \rightarrow U$ when $\lambda \rightarrow 1$. This means that the span condition is not satisfied, which should be no surprise as there are certain relations between the vectors in $\left[P_{-1}, Q_{0}\right]$. For instance one can easily check that $[p, q]+\left[p^{\prime}, q^{\prime}\right]=0$.

There are in fact two other 1-parameter families of complex Hadamard matrices containing $U$, which can be obtained by noticing that $U$ has certain symmetries. Indeed, let $\sigma_{1}, \sigma_{2}$ denote the following 
permutation matrices:

$$
\sigma_{1}=\left(\begin{array}{ccccccc}
1 & 0 & 0 & 0 & 0 & 0 & 0 \\
0 & 0 & 0 & 0 & 0 & 0 & 1 \\
0 & 0 & 1 & 0 & 0 & 0 & 0 \\
0 & 0 & 0 & 1 & 0 & 0 & 0 \\
0 & 0 & 0 & 0 & 0 & 1 & 0 \\
0 & 0 & 0 & 0 & 1 & 0 & 0 \\
0 & 1 & 0 & 0 & 0 & 0 & 0
\end{array}\right), \sigma_{2}=\left(\begin{array}{ccccccc}
1 & 0 & 0 & 0 & 0 & 0 & 0 \\
0 & 1 & 0 & 0 & 0 & 0 & 0 \\
0 & 0 & 0 & 0 & 0 & 0 & 1 \\
0 & 0 & 0 & 0 & 0 & 1 & 0 \\
0 & 0 & 0 & 0 & 1 & 0 & 0 \\
0 & 0 & 0 & 1 & 0 & 0 & 0 \\
0 & 0 & 1 & 0 & 0 & 0 & 0
\end{array}\right)
$$

It is easy to check that $\sigma_{1} U \sigma_{2}^{*}=U$ Thus $\tilde{U}(\lambda)=\sigma_{1} U(\lambda) \sigma_{2}^{*}$ also yields a continuous parametric family of commuting squares containing $\mathcal{C}$, as $\tilde{U}(1)=U$. Similarly one can construct another family by using $\sigma_{2} U \sigma_{1}^{*}=U$

Let $h$ denote the direction of the family of commuting squares obtained from $U(\lambda)$. Since $U(\lambda)=\left(I+(\lambda-1) p q+(\bar{\lambda}-1) p^{\prime} q^{\prime}\right) U$, it easily follows that $h=\frac{p q-p^{\prime} q^{\prime}}{\left\|p q-p^{\prime} q^{\prime}\right\|}$. Let $\tilde{h}$ denote the direction of the family $\tilde{U}(\lambda)$. Since $\tilde{U}(\lambda)=\sigma_{1} U(\lambda) \sigma_{2}^{*}$, we have $\tilde{h}=\sigma_{1} h U \sigma_{2}^{*} U^{*}=\sigma_{1} h \sigma_{1}^{*}$. It is easy to check that $h \neq \tilde{h}$.

Clearly for both $h$ and $\tilde{h}$ the conditions of Theorem 3.1 must be satisfied, and thus the system (5) must be consistent. However, let $h_{0}=\frac{h+\tilde{h}}{\|h+\tilde{h}\|}$. It is an easy check with Mathematica that the system (5) is not consistent, with $h_{0}$ instead of $h$.

Thus, $h_{0}$ is orthogonal to $H(\mathfrak{C})$ but it is not a direction of convergence. It is in fact not surprising that for an arbitrary linear combination of $h, \tilde{h}$ the conditions of Theorem 3.1 are no longer satisfied, as the map $h \rightarrow \tau\left(p q h^{2}\right)-\tau(p h q h$ ) is no longer linear (for fixed $p, q)$ ). Our choice of $h_{0}$ is unimportant, since it is immediate to see that any linear combination of $h, \tilde{h}$ with non-zero coefficients yields a consistent system (5) if and only if one such combination yields a consistent system.

We conclude with the following corollary, asserting that there exists no multi-parametric $C^{1}$ family of complex Hadamard matrices containing both families $U(\lambda), \tilde{U}(\lambda)$. Such questions are of interest in the classification of complex Hadamard matrices.

Corollary 4.1. There does not exist a differentiable map $W: \mathbb{T} \times \mathbb{T} \rightarrow$ $M_{7}(\mathbb{C})$, taking values in the set of complex Hadamard matrices and satisfying $W(\lambda, 1)=\sqrt{7} U(\lambda)$ and $W(1, \lambda)=\sqrt{7} \tilde{U}(\lambda)$ for all $\lambda \in \mathbb{T}$ close to 1 .

Proof. The derivative of the map $\lambda \rightarrow \frac{1}{\sqrt{7}} W(\lambda, \lambda) U^{*}$ at $\lambda=1$ equals $h+\tilde{h}$. It follows that the (normalized) direction of convergence of the 
unitaries $\frac{1}{\sqrt{7}} W(\lambda, \lambda) U^{*}$ is $\frac{h+\tilde{h}}{\|h+\tilde{h}\|}=h_{0}$. However, we have seen that $h_{0}$ is not a direction of convergence for $\mathfrak{C}$, contradiction.

\section{REFERENCES}

[Chr] E.Christensen, Subalgebras of a finite algebra, Mathematische Annalen 243, 17-29 (1979)

[H] U.Haagerup, Orthogonal maximal abelian *-subalgebras of the $n \times n$ matrices and cyclic n-roots, Operator Algebras and Quantum Field Theory (ed. S.Doplicher et al.), International Press (1997),296-322

[Jon] V.F.R.Jones, Index for subfactors, Invent. Math 72 (1983), 1-25.

[JS] V.F.R.Jones and V.S.Sunder, Introduction to subfactors, London Math. Soc. Lecture Notes Series 234, Cambridge University Press, 1997.

[Ni] R. Nicoara, A finiteness result for commuting squares of matrix algebras, $J$. Operator Theory 55 (2006), 295-310.

[Pe] M.Petrescu, Existence of continuous families of complex Hadamard matrices of certain prime dimensions and related results, $\mathrm{PhD}$ thesis, University of California Los Angeles, 1997.

[Po1] S.Popa, Classification of subfactors : the reduction to commuting squares, Invent. Math., 101(1990),19-43

[Po2] S.Popa, Classification of amenable subfactors of type II, Acta Mathematica, 172, 163-255 (1994).

[Po3] S.Popa, Othogonal pairs of *-subalgebras in finite von Neumann algebras, J. Operator Theory 9, 253-268 (1983)

[TaZy] W. Tadej and K. Zyczkowski, A concise guide to complex Hadamard matrices Open Systems \& Infor. Dyn.,13(2006), 133-177, quant-ph/0512154 